\title{
Effets de la gouvernance sur les ressources naturelles: évidences en Afrique subsaharienne
}

\author{
DIEME $^{1}$ Madaniou \\ Laboratoire de recherches économiques et monétaires (LAREM) \\ Ecole Doctorale Développement Durable et Société (ED2DS) \\ Université de Thiès \\ Thiès - Sénégal
}

\begin{abstract}
Résumé : Ce papier a pour but de revisiter la relation entre recettes issues des ressources naturelles et PIB par habitant en ASS, à l'aide d'un modèle de données de panel à effets de seuil, modèle de type Threshold Auto Regressive (TAR), inspiré des travaux de Hansen (1999). Les données couvrent 37 pays sur 48 de l'ASS et s'étendent sur la période 2002-2019. Les résultats montrent que sur la période, les rentes des ressources naturelles ont faiblement, négativement et significativement contribué à l'amélioration du bien-être des populations d'ASS. Les estimations montrent que l'élasticité entre les rentes des ressources naturelles et le PIB par habitant est comprise entre -0,093 et -0,079.

Au même moment, les indicateurs de gouvernance affichent de faibles liaisons avec le PIB par habitant, contribuant faiblement à l'amélioration du niveau de vie des populations de l'ASS, quoique de façon positive sur la période. Cependant, seules les contributions de la Qualité de la réglementation et de l'Etat de droit ont été significatives. En outre, les résultats montrent qu'il n'y a pas de différences significatives entre les pays d'ASS avec de faibles institutions et ceux avec de meilleures institutions.

Le modèle à effets de seuil unique validé entre les rentes des ressources naturelles et le développement estime un seuil à $3,14 \%$. Les résultats montrent que lorsque les rentes des ressources naturelles sont inférieures à ce seuil, on est en présence d'une relation positive entre les ressources naturelles et le PIB par habitant (élasticité de $0,05 \%$ ), alors que lorsqu'elles sont supérieures à ce seuil, la relation est négative $(-0,08 \%)$.
\end{abstract}

Mots-clés : ressources naturelles ; développement ; institutions ; modèle à effets de seuil.

Digital Object Identifier (DOI): https://doi.org/10.52502/ijesm.v1i3.197

${ }^{1}$ Doctorant à l'Université de Thiès. Sénégal. 


\section{Introduction}

Les ressources naturelles, source de richesse, ont la capacité d'accroître la prospérité ou d'entraver la croissance économique (Moshiri et Hayati, 2017). Elles ont la capacité de doper la croissance économique et de stimuler son caractère inclusif en sortant beaucoup de populations de la pauvreté. Selon Baldé (2020), 26 sur 48 pays d'Afrique subsaharienne dépendent de leurs dotations en ressources naturelles et que, à l'horizon 2030, un peu plus de 6 pauvres sur 10 résideront dans ces pays subsahariens riches en ressources, contre un peu plus de 1 pauvre sur 10 dans les années 2000. Ainsi, bien exploiter les ressources naturelles pour créer suffisamment d'emplois, promouvoir la croissance économique et améliorer le développement dans les pays subsahariens riches en ressources naturelles est un défi majeur pour sortir des millions de personnes de la pauvreté (Baldé, 2020).

Le secteur des ressources naturelles reste confronté à des problèmes tels que volatilité des prix, ressources épuisables, incertitude et concurrence exercée par les énergies dites propres et renouvelables. Ces contraintes sont renforcées par la mise en œuvre de politiques économiques pas ou peu adaptées et, par la suite, le secteur est miné par la corruption et les conflits. Ainsi, les ressources naturelles se présentent souvent comme des problèmes pour les pays qui en sont bien dotés.

Les résultats présentés dans le chapitre 1 montrent que les institutions ont un impact positif sur le PIB par habitant dans les pays riches en ressources naturelles (PRRN). Dans ces pays, le niveau de richesse des populations peut être positivement et significativement impacté par (i) les efforts consentis par les gouvernements pour lutter contre la corruption, (ii) le respect des règles et administrations de la société et la confiance des acteurs à ces règles, (iii) la stabilité politique et l'absence de violence / terrorisme et (iv) la qualité de la réglementation. Tous ces aspects de gouvernance peuvent jouer un rôle important dans le processus de développement dans les PRRN.

Cependant, la qualité des services publics, celle de la fonction publique, celle de la formulation et de la mise en œuvre des politiques, le degré de son indépendance vis-à-vis des pressions politiques et la crédibilité de l'engagement du gouvernement à l'égard de ces politiques n'auraient pas produit des effets significatifs sur le revenu par tête des populations des PRRN. Aussi, la participation des populations aux affaires publiques, leur liberté d'expression et d'association, de même que l'existence de médias libres et indépendants, n'impactent-elles pas le revenu par habitant de ces populations.

Malgré leur importance, ces résultats globaux ne donnent que peu d'indications sur les pays d'Afrique subsaharienne. La nature de la relation entre ressources naturelles, gouvernance et développement, pour les pays de cette zone, reste inconnu. Des tendances lourdes montrent que plusieurs pays d'Afrique subsaharienne présentent des symptômes de maladie hollandaise qui vont avec de faibles capacités institutionnelles et managériales.

Au cours de ces dernières décennies, des pays d'Afrique subsaharienne ont démarré l'exploitation de ressources naturelles, venant s'ajouter à la liste de pays déjà producteurs. D'autres pays intégreront bientôt cette catégorie. Cette situation traduit l'importance grandissante des ressources naturelles dans les pays d'Afrique au sud du Sahara.

Ce papier a pour but de revisiter la relation entre recettes issues des ressources naturelles et PIB par habitant en ASS, à l'aide d'un modèle de données de panel à effets de seuil, modèle de type Threshold Auto Regressive (TAR), inspiré des travaux de Hansen (1999).

Sous l'hypothèse principale selon laquelle les effets des ressources naturelles sur le développement dépendent de l'état de la gouvernance, plusieurs questions peuvent se poser : (i) comment les ressources naturelles affectent le développement en ASS ? (ii) Y a-t-il des différences entre les pays présentant de bons indices de gouvernance et ceux présentant de mauvais indices? (iii) Les effets des ressources 
naturelles sur le développement en ASS sont-ils différentiés entre les PRRN et ceux pauvres en ressources naturelles ? (iv) Qu'en est-il au sein de chacun de ces groupes de pays?

Pour répondre à ces interrogations, le présent chapitre est organisé comme suit : la deuxième section présente une brève revue de la littérature ; la troisième section aborde la méthodologie et les données du modèle ; la quatrième section donne les résultats du modèle et la cinquième section est consacrée à la discussion et la dernière section à la conclusion.

\section{Revue de la littérature}

Au cours de ces dernières années, plusieurs auteurs ont rejeté la spécification linéaire dans la relation entre ressources naturelles et développement, en adoptant celle à effet de seuil par exemple (Sarmidi et al., 2011 ; Hayat \& Tahir, 2017 ; Moshiri \& Hayati, 2017 ; Khan et al., 2020). Ces auteurs ont cherché à estimer les effets non linéaires des ressources naturelles sur le développement. En outre, ces types de modèle permettent de déterminer des seuils de ressources naturelles au-delà desquels un changement de régime est opéré.

Les principaux résultats apportent des preuves empiriques qui ont montré que les ressources naturelles pouvaient entraver ou doper le développement au-delà d'un certain seuil.

Moshiri et Hayati (2017) ont développé un modèle de croissance prenant en considération les ressources naturelles afin d'estimer l'effet de la dépendance aux ressources naturelles sur la croissance économique. Ils ont utilisé différentes mesures des ressources naturelles et en contrôlant la qualité des institutions dans 149 pays sur la période 1996-2010. Leurs résultats montrent que l'abondance des ressources naturelles a un effet positif et significatif sur la croissance du PIB. Hayat et Tahir (2017) ont analysé le lien entre ressources naturelles, IDE et croissance économique, en utilisant un modèle de seuil à effet fixe avec des données de panel annuelles pour 83 pays. Leurs résultats montrent que les IDE ont un fort impact positif sur la croissance économique du pays d'accueil lorsque les exportations de ressources naturelles du pays d'accueil sont inférieures au seuil estimé statistiquement significatif, avec cependant, une croissance économique plus faible si les exportations de ressources naturelles du pays sont supérieures au seuil estimé.

De façon empirique, Khan et al. (2020) ont examiné les liens entre la rente des ressources naturelles et le développement financier pour le Pakistan sur la période 1984-2018. Ils ont abouti aux résultats selon lesquels la rente des ressources naturelles influence négativement le développement financier, au moment où la qualité institutionnelle le stimule, atténuant ainsi la relation. Aussi, les auteurs ont-ils déterminé une seule valeur seuil significative de 3,097 au-dessus de laquelle, la relation rente-financement des ressources devient non linéaire : un premier régime (jusqu'à ce seuil) caractérisé par un coefficient de 3,228 qui diminue légèrement jusqu'à 2,804 pour le second régime (au-dessus du niveau seuil).

Des différences de seuil sont notées entre les pays en fonction de leurs spécificités. Selon Kelikume (2018), la valeur du seuil des ressources naturelles pour les pays riches en ressources était plus importante que celle des autres pays : 12,5\% contre 9,4\%. Il est à noter que les ressources naturelles ont eu un impact positif sur le développement pour des seuils de ressources naturelles inférieures à ces valeurs pour les pays africains. Cependant, lorsque les ressources naturelles ont dépassé ces seuils, elles ont eu un effet négatif sur le développement.

Khan et al. (2020) ont examiné les liens empiriques entre la rente des ressources naturelles et le développement financier pour le Pakistan, entre 1984 et 2018, en prenant en compte le rôle important de la qualité institutionnelle.

Epo et Nochi Faha (2019) ont examiné le rôle des institutions dans la relation entre les ressources naturelles et la croissance économique à l'aide d'un panel de données de 44 pays africains sur la période 
1996-2016. Pour Moshiri et Hayati (2017), l'impact de la dépendance aux ressources naturelles sur la croissance du PIB dépend du type de ressources naturelles et de la qualité des institutions. Les résultats de Sarmidi et al. (2011) mettent en évidence le fait que les nations qui ont une faible qualité institutionnelle dépendent beaucoup des ressources naturelles tandis que les pays avec des institutions de haute qualité sont relativement moins dépendants des ressources naturelles pour générer de la croissance. Ces résultats sont confirmés par Clootens et Kirat (2020), qui ont montré que dans les pays à revenu élevé (souvent à haute qualité institutionnelle), les ressources naturelles ne jouent qu'un rôle mineur dans l'explication des différences de taux de croissance nationaux. Au contraire, dans les pays à faible revenu (souvent à faible qualité institutionnelle), l'abondance semble être une bénédiction mais la dépendance limite la croissance.

Les résultats de Epo et Nochi Faha (2019) montrent que, d'une part, la relation entre les ressources naturelles et la croissance économique varie pour les indicateurs de qualité institutionnelle et la mesure des ressources naturelles et, d'autre part, la relation non linéaire entre les ressources naturelles et la croissance économique est considérablement améliorée lorsque l'on considère les variables état de droit et qualité de la réglementation. Les résultats de Moshiri et Hayati (2017) montrent que parmi les différents indices utilisés pour les qualités institutionnelles, l'efficacité du gouvernement, la qualité de la réglementation et l'état de droit sont plus efficaces pour éviter l'effet négatif de la dépendance aux ressources.

De même, les travaux empiriques de Sarmidi et al. (2011) révèlent qu'il existe un effet de seuil dans la relation ressources naturelles - croissance économique. En utilisant une technique innovante d'estimation de seuil, ils ont constaté que l'impact des ressources naturelles n'est significatif pour la croissance économique qu'après avoir atteint un certain seuil de qualité institutionnelle. Quant à Moshiri et Hayati (2017), ils ont estimé les seuils au-dessus desquels différents types de qualités institutionnelles peuvent transformer une malédiction en une bénédiction pour différents types de dépendance aux ressources naturelles.

Les résultats de Khan et al. (2020) ont montré que la rente des ressources naturelles influence négativement le développement financier, tandis que la qualité institutionnelle stimule le développement financier et modère positivement la relation dans le contexte du Pakistan. Ainsi, Khan et al. (2020) ont trouvé une seule valeur seuil significative des institutions de 3,097 au-dessus de laquelle la relation rentefinancement des ressources devient non linéaire, car jusqu'à ce seuil, le coefficient est de 3,228, qui diminue légèrement à 2,804 au-dessus du niveau seuil.

\section{Méthodologie}

\subsection{Spécification du modèle}

Ce chapitre examine les non-linéarités dans la relation entre les ressources naturelles et le développement en ASS. Son objectif est d'apporter des éclairages sur les effets non linéaires et les seuils des ressources naturelles sur le développement à long terme en ASS. Clootens et Kirat (2020) ont rejeté le modèle linéaire, couramment utilisé dans les régressions de croissance, en faveur d'une alternative à régimes multiples dans leur analyse du comportement des taux de croissance entre les pays en ce qui concerne l'abondance et la dépendance des ressources. Ils ont constaté que les pays présentaient des comportements différents vis-à-vis des ressources naturelles en fonction de leur niveau de développement initial.

Dans un premier temps, des seuils sont utilisés de façon délibérée. Un pays est considéré comme riche en ressources naturelles, si la part des rentes issues de ces dernières est au moins égale à $10 \%$ de son PIB. Un pays est considéré avoir de bonnes institutions, s'il se trouve dans la moitié des pays les mieux classé au sein de l'échantillon. 
Au premier niveau d'analyse, un modèle global est testé pour tous les pays de l'ASS, afin de déterminer les effets des ressources naturelles sur le développement. Au deuxième niveau, le même test est effectué pour les pays riches en ressources naturelles et les autres pays d'ASS, dans l'optique de déceler les potentielles écarts entre ces deux groupes de pays. Dans tous les cas, les différences de niveau de gouvernance sont prises en compte.

Dans ce contexte, un modèle économétrique est développé pour déterminer de manière empirique, la manière dont les recettes issues des ressources naturelles affectent ou non le PIB par habitant dans les pays d'Afrique subsaharienne, en utilisant des données de panel de $37^{2}$ pays sur 48 de l'ASS. Pour réaliser ce test, un modèle à effets de seuil, inspiré de celui de Hansen (1999), a été employé sur la période 20022019.

Les recettes issues des ressources naturelles ont été retenu comme seule variable de seuil dans ce modèle. En utilisant ce ratio, comme proxy de la richesse en ressources naturelles, ce chapitre cherche à vérifier dans le cadre d'un modèle de régression à effets de seuil, si les recettes issues des ressources naturelles engendrent ou non du développement, avec comme proxy le PIB par habitant, en ASS ; autrement dit, si l'hypothèse de la malédiction des ressources est confirmée ou non.

Dans un second temps, les seuils sont déterminés avec le modèle. Une régression de seuil de panel dynamique est appliquée, pour les 37 pays africains pour la période 2002-2019, afin de tenir compte du biais d'endogénéité potentiel dans le modèle. L'échantillon a ensuite été divisé entre pays riches en ressources et pays non riches en ressources, afin de voir s'il existe des différences dans le lien empirique entre ressources naturelles et développement à long terme.

Une modélisation en termes d'effet de seuil est retenue dans cette étude. Pour ce faire, elle fait l'hypothèse que, l'impact des ressources naturelles sur le développement est différent selon un niveau donné atteint par les institutions. Ce faisant, ce papier emploie le modèle de régression de seuil, de type Threshold Auto Regressive (TAR) proposé par Hansen (1999) pour analyser la corrélation entre ressources naturelles, développement et autres variables économiques dans les 37 pays d'ASS, en utilisant comme variable de seuil, les institutions et comme variable régime-dépendant, les recettes issues des ressources naturelles. Il s'agit d'un modèle de panel à effet de seuil fixe (fixed-effect panel threshold model). La méthode bootstrap est également utilisée pour tester la signification statistique de l'effet seuil. Ce modèle à la possibilité de présenter plusieurs régimes distincts. Le modèle pour un seuil unique s'exprime comme suit : Yit $=\mu+\operatorname{Xit}($ qit $<\gamma) \beta 1+\operatorname{Xit}($ qit $\geq \gamma) ß 2+$ ui + eit

où Yit est la variable dépendante, Xit le vecteur des variables explicatives, qit la variable seuil, $\gamma$ est le paramètre de seuil qui divise l'équation en deux régimes, avec les coefficients $\beta 1$ et $\beta 2$. Le paramètre ui exprime l'effet individuel tandis que eit est le terme d'erreur.

Enfin, les mêmes estimations sont réalisées en prenant cette fois-ci les institutions comme variable régime-dépendant et les recettes issues des ressources naturelles comme variable de seuil.

\subsection{Données du modèle}

Des données annuelles d'un panel de 37 pays d'ASS ont été utilisées sur la période 2002-2019 pour analyser la relation entre ressources naturelles et développement. La variable dépendante est le PIB par habitant (pibpc). C'est l'un des indicateurs les plus utilisés pour analyser le développement. La variable régime-dépendant est les rentes tirées des ressources naturelles (ress). La principale variable d'intérêt, est la variable de seuil, c'est à dire les institutions. Les variables de contrôle comprennent les investissements (invest)et les exportations (exports).

\footnotetext{
28 pays ont été écartés de l'étude, en raison de données statistiques incomplètes.
} 
Toutes ces variables sont transformées en logarithme afin de pouvoir mieux interpréter les résultats. Les données sont extraites des bases de données de la Banque mondiale : World Development Indicators (WDI) et World Governance Indicators (WGI).

Le contrôle de la corruption capture les perceptions de la mesure dans laquelle le pouvoir public est exercé à des fins privées, y compris les petites et les grandes formes de corruption, ainsi que la « capture » de l'État par les élites et les intérêts privés.

L'efficacité des pouvoirs publics reflète les perceptions de la qualité des services publics, de la qualité de la fonction publique et du degré de son indépendance vis-à-vis des pressions politiques, de la qualité de la formulation et de la mise en œuvre des politiques et de la crédibilité de l'engagement du gouvernement à l'égard de ces politiques.

La stabilité politique et l'absence de violence / terrorisme mesurent les perceptions de la probabilité d'instabilité politique et / ou de violence à motivation politique, y compris le terrorisme.

L'état de droit saisit la façon dont les agents ont confiance et respectent les règles de la société, et en particulier la qualité de l'exécution des contrats, les droits de propriété, la police et les tribunaux, ainsi que la probabilité de criminalité et de violence.

La qualité de la réglementation reflète les perceptions de la capacité du gouvernement à formuler et à mettre en œuvre des politiques et des réglementations solides qui permettent et favorisent le développement du secteur privé.

La voix et la responsabilité saisissent les perceptions de la mesure dans laquelle les citoyens d'un pays peuvent participer à la sélection de leur gouvernement, ainsi que la liberté d'expression, la liberté d'association et des médias libres.

Source : Banque mondiale, Indicateurs de gouvernance dans le monde

\section{Résultats}

Le tableau ci-dessous est le résultat d'un panel équilibré de 666 observations. Le PIB moyen par habitant, en logarithme, dans les 37 pays d'ASS retenus, est de 6,952 \$, moyenne comprise entre 4,732 et 9,324. Le ratio rentes des ressources naturelles/PIB, est en moyenne de 1,9 en logarithme ; il s'échelonne entre $-6,750$ et 4,072. Il est constaté que les ressources, en compagnie des investissements et des exportations, sont les plus volatiles parmi les variables.

Tableau 1 : Statistiques descriptives

\begin{tabular}{|c|c|c|c|c|c|}
\hline Variables & Observations & Moyennes & Ecart type & Minimum & Maximum \\
\hline lpibpc & 666 & 6,952 & 0,976 & 4,732 & 9,324 \\
\hline linvest & 666 & 21,396 & 1,585 & 17,235 & 25,427 \\
\hline lexports & 666 & 21,645 & 1,617 & 17,444 & 25,691 \\
\hline lress & 666 & 1,900 & 1,509 & $-6,750$ & 4,072 \\
\hline lvar & 666 & 3,256 & 0,693 & 0,901 & 4,324 \\
\hline lpvr & 666 & 3,077 & 0,989 & $-0,742$ & 4,541 \\
\hline lger & 666 & 2,885 & 0,959 & $-0,054$ & 4,403 \\
\hline lrqr & 666 & 3,136 & 0,826 & $-0,713$ & 4,427 \\
\hline lrlr & 666 & 2,966 & 0,995 & $-0,747$ & 4,427 \\
\hline lccr & 666 & 3,020 & 0,907 & $-0,683$ & 4,441 \\
\hline
\end{tabular}




\section{Estimations pour tous les pays}

Quelle que soit la variable institutionnelle retenue, les rentes des ressources naturelles ont faiblement, négativement et significativement contribué à l'amélioration du bien-être des populations d'ASS sur la période 2002-2019. Les estimations montrent que l'élasticité entre les rentes des ressources naturelles et le PIB par habitant est comprise entre $-0,093$ et $-0,079$ en ASS. Une augmentation des rentes des ressources naturelles se traduisent par de minimes pertes de bien-être pour les populations de l'ASS.

Au même moment, les indicateurs de gouvernance affichent de faibles liaisons avec le PIB par habitant, contribuant faiblement à l'amélioration du niveau de vie des populations de l'ASS, quoique de façon positive sur la période. Cependant, seules les contributions de la Qualité de la réglementation (lrqr) et de l'Etat de droit (lrlr) ont été significatives.

\section{Estimations pour tous les pays par niveau d'institutions}

Les résultats montrent qu'il n'y a pas de différences significatives entre les pays d'ASS avec de faibles institutions et ceux avec de meilleures institutions : les résultats épousent généralement ceux obtenus au niveau global. Bien que la contribution des rentes des ressources naturelles au PIB par habitant soit négative, pour les pays d'ASS, entre 2002 et 2019, l'intensité est plus forte dans les pays d'ASS caractérisés par de plus faibles institutions. Dans les deux groupes, l'Etat de droit (lrlr) s'est montré significatif alors que la Qualité de la réglementation (lrqr) est significative dans les pays d'ASS à faible qualité institutionnelle et l'Efficacité des pouvoirs publics (lger) est significative dans les pays d'ASS à forte qualité institutionnelle.

\section{Estimations pour tous les pays par niveau de ressources}

Les ressources naturelles contribuent négativement au PIB par habitant, quel que soit le niveau de richesse en ressources. Cependant, cette contribution négative n'est pas significative dans les pays riches en ressources naturelles. De même, l'impact est plus faible dans les pays riches en ressources naturelles. Par ailleurs, dans les pays pauvres en ressources, seuls l'Etat de droit (lrlr) et le Contrôle de la corruption (lccr) sont ont été significatifs pour le PIB par habitant, avec une contribution positive pour le premier cité (lrlr) et négative pour le second (lccr). Dans les pays riches en ressources, les variables institutionnelles ont eu une contribution positive, mais seules celles relatives à la Qualité de la réglementation (lrqr), l'Etat de droit (lrlr) et le Contrôle de la corruption (lccr) ont été significatives.

\section{Estimations pour tous les pays par niveau de ressources et d'institutions}

Dans les Pays Pauvres en Ressources Naturelles avec de Faibles Institutions, les ressources naturelles ont eu un impact négatif sur le PIB par habitant, estimé dans la fourchette de [-0,292 et -0,0956]. Cette fourchette est de [-0,081 et $-0,054]$ dans les Pays Pauvres en Ressources Naturelles avec de Fortes Institutions. Elle est estimée à $[-0,066$ et 0,011$]$ et $[-0,128$ et 0,056$]$ respectivement dans les Pays Riches en Ressources Naturelles avec de Faibles Institutions et dans les Pays Riches en Ressources Naturelles avec de Fortes Institutions. Dans ces deux derniers groupes, les élasticités estimées ne sont pas significatives.

Dans les Pays Pauvres en Ressources Naturelles avec de Faibles Institutions, la seule variable institutionnelle significative est l'Etat de droit (lrlr); dans les Pays Pauvres en Ressources Naturelles avec de Fortes Institutions, les variables institutionnelles significatives sont l'Etat de droit (lrlr) et le Contrôle de la corruption (lccr) ; dans les Pays Riches en Ressources Naturelles avec de Faibles Institutions, les variables institutionnelles significatives sont la Voix citoyenne et responsabilité (lvar), la Qualité de la réglementation (lrqr) et l'Etat de droit (lrlr) ; dans les Pays Riches en Ressources Naturelles avec de Fortes Institutions, toutes les variables institutionnelles sont significatives sauf l'Etat de droit (lrlr). 
Tableau 2 : Estimations avec des seuils fixés de façon délibérée

\begin{tabular}{|c|c|c|c|c|c|c|c|c|c|c|c|}
\hline \multirow{2}{*}{\multicolumn{12}{|c|}{ Légende $: * p<.05 ; * * p<.01 ; * * * p<.001$}} \\
\hline & & & & & Estimation & & & & & & \\
\hline lress &, $09325374 * * *$ & lress &, 09218537 *** & lress & $-0,09279682 * * *$ & lress & $-0,08887006$ **** & 1ress & $-0,07880047$ **** & lress & $-0,0928332^{* * * *}$ \\
\hline linvest & $0,27706634 * * *$ & linvest & $0,27233263 * * *$ & linvest & $0,27594341^{* * * *}$ & linvest & $0,27029453 * * *$ & linvest & $0,25265669^{*} * *$ & linvest & $0,27303514 * * *$ \\
\hline lexports & $0,3077111^{* * * *}$ & lexports & $0,31066078^{* * * *}$ & lexports & $0,30896505^{* * * *}$ & lexports & $0,3107333^{* * * *}$ & lexports & $0,32669103 * * *$ & lexports & $0,3130333 * * *$ \\
\hline lvar & 0,00214517 & lpvr & 0,02412868 & lger & 0,00701842 & |lrqr & $0,07814119^{* * * *}$ & Irlr & $0,08910482^{* * *}$ & lecr & 0,02981549 \\
\hline _cons & $-5.4658676 * * *$ & _cons & $-5.4977131^{* * *}$ & cons & $-5.4831144 * * *$ & _cons & $-5.6327784 * * *$ & _cons & $-5.6391659 * * *$ & _cons & $-5.578681 * * *$ \\
\hline \multirow{2}{*}{\multicolumn{12}{|c|}{$\begin{array}{l}\text { Estimations pour tous les pays par niveau d'institutions } \\
\text { Faibles institutions }\end{array}$}} \\
\hline & & & & & & & & & & & \\
\hline Iress & $-0,08684037^{* *}$ & Iress & $-0,15477847 * * *$ & lress & $-0,12366425 * * *$ & lress & $-0,11172813 * * * *$ & lress & $-0,10448027^{* * * *}$ & Iress & $-0,15270996 * * *$ \\
\hline linvest & $0,24858775^{* * * *}$ & linvest & $0,28276199^{* * *}$ & linvest & $0,26659233^{* * * *}$ & linvest & $0,27084476^{* * * *}$ & linvest & $0,21808461 * * *$ & linvest & $0,23639007 * * *$ \\
\hline lexports & $0,34692421 * * * *$ & lexports & $0,2995449 * * *$ & lexports & $0,30956642 * * * *$ & lexports & $0,31007467^{* * * *}$ & lexports & $0,36708576 * * *$ & lexports & $0,37569498 * * * *$ \\
\hline lvar & $-0,03621364$ & lpvr & $-0,02227793$ & lger & $-0,00661574$ & lrqr & $0,08328528^{* * *}$ & Irlr & $0,08460286 * * *$ & lecr & 0,0101388 \\
\hline _cons & $-5,6271519^{* * * *}$ & _cons & $-5,4280929 * * *$ & con & $-5,2197596 * * *$ & _cons & $-5,5969866 * * *$ & _co & $-5,800635 * * *$ & cons & $731^{* * * *}$ \\
\hline \multicolumn{12}{|c|}{ Fortes institutions } \\
\hline Iress & $-0,0949729^{* * * *}$ & Iress & $-0,04821538^{* * *}$ & Iress & $-0,08025847 * * * *$ & Iress & $-0,08464118^{* * * *}$ & Iress & $-0,06173194 * * * *$ & Iress & $-0,05664717 * *$ \\
\hline linvest & $0,30148864 * * *$ & linvest & $0,22736972 * * *$ & linvest & $0,29699323 * * * *$ & linvest & $0,26787743 * * *$ & linvest & $0,3151343^{* * * *}$ & linvest & $0,31328335 * * *$ \\
\hline lexports & $0,26330722 * * *$ & lexports & $0,33760792 * * *$ & lexports & $0,293902 * * *$ & lexports & $0,32068198 * * * *$ & lexports & $0,26456121 * * *$ & lexports & 0,25218923 **** \\
\hline lvar & & lpvr & & lger & & \begin{tabular}{|l|l|}
$\operatorname{lrgr}$ \\
\end{tabular} & & Irlr & $701 * * *$ & Iccr & $-0,02107892$ \\
\hline cons & $-5,4308576^{* * * *}$ & _cons & $-5,1210249 * * *$ & cons & $-6,0016996 * * *$ & _cons & $-5,7939727 * * * *$ & _cons & $-5,8193709 * * *$ & _cons & $-4,91774 * * *$ \\
\hline \multirow{2}{*}{\multicolumn{12}{|c|}{$\begin{array}{l}\text { Estimations pour tous les pays par niveau de ressources naturelles } \\
\text { Pauvres en ressources }\end{array}$}} \\
\hline & & & & & & & & & & & \\
\hline lress & $-0,08735879 * * * *$ & Iress & $-0,0884104 * * *$ & lress & $-0,08716095 * * *$ & lress & $-0,08909194 * * * *$ & Iress & $-0,07554288^{* * * *}$ & lress & $-0,09247084 * * *$ \\
\hline linvest & $0,34592708 * * * *$ & linvest & $63^{* * * * *}$ & linvest & $0,3458658^{* * * *}$ & linvest & $0,35045052^{* * * *}$ & linvest & $0,30835332 * * *$ & linvest & $0,35829479^{* * * *}$ \\
\hline lexports & 0,209 & lexport & & lexpo & $2 * * *$ & lexpo & $3 * * *$ & $\operatorname{lexp}$ & $27 * * *$ & lexports & $15889 * * *$ \\
\hline lvar & 0,00136298 & lpvr & & lger & 0,00214949 & lrgr & $-0,02375129$ & Irlr & $0,09793124 * * *$ & lccr & $-0,05188677^{*}$ \\
\hline _cons & $-4,8652909 * * *$ & cons & $-4,9391892 * * *$ & cons & $-4,8727146 * * * *$ & cons & $6^{* * * * *}$ & _cons & $176 * * *$ & cons & $-4,6401798^{* * * *}$ \\
\hline \multicolumn{12}{|c|}{ Riches en ressources } \\
\hline Iress & $-0,04832626$ & Iress & & $\operatorname{lr}$ & 74924 & lress & 58 & lre & 24 & lress & 01579 \\
\hline linvest & $* * *$ & linvest & & linvest & $764 * * *$ & linvest & 0,21 & lit & $6 * * *$ & linvest & $0,22167953 * * *$ \\
\hline lexports & $5 * * *$ & lexports & **** & $\operatorname{lexpo}$ & $4^{* * * * *}$ & $\begin{array}{ll}\operatorname{lexpc} \\
\end{array}$ & & $\operatorname{lexp}$ & $6 * * *$ & lexports & $0,40559235^{* * * *}$ \\
\hline lvar & 614 & lpvr & & lger & 21 & lrgr & $8 * * *$ & Irlr & $223^{* *}$ & lccr & $0,07611439 * * *$ \\
\hline _cons & $-6,661387 * * *$ & cons & $-6,5506059 * * *$ & cons & $-6,7060542 * * *$ & con & $-6,8926297 * * *$ & cons & $-6,6991565 * * *$ & cons & $-6,9202928 * * *$ \\
\hline \multicolumn{12}{|c|}{ PPRN et Faibles institutions } \\
\hline Iress & $88^{* *}$ & Iress & $166^{* * * * *}$ & Iress & $-0,18524292^{* * *}$ & Iress & $-0,22489073 * *$ & Ire: & $-0,0955228$ & Iress & $-0,29198471^{* * * *}$ \\
\hline linvest & $* * *$ & linvest & & linvest & $0,32625411^{* * * *}$ & linvest & $9 * * *$ & & $801 * * *$ & linvest & 7722 **** \\
\hline lexports & 0,25 & lexpo & & le & & $\operatorname{lexp}$ & & $\operatorname{lexp}$ & $49 * * *$ & $\operatorname{lexpo}$ & $435 * *$ \\
\hline lvar & & lpvr & & lger & $-0,8 \quad r \quad r$ & lrqr & & 1rlr & $451 * *$ & lccr & $-0,0880318$ \\
\hline _cons & $-4,8615124 * * *$ & cons & $-4,5392362 * * *$ & cons & $-3,4616315 * * *$ & & $-4,3052544 * * *$ & cons & $-4,3636603 * * *$ & cons & $-3,7628742 * * *$ \\
\hline \multicolumn{12}{|c|}{ PPRN et Fortes institutions } \\
\hline Iress & $27 * * * *$ & Iress & $62^{* * *}$ & Iress & $-0,07670716^{* * * * *}$ & lress & $44 * * *$ & lress & $6334 * * *$ & lress & $-0,06518264 * * *$ \\
\hline linvest & $7 * * *$ & linves & & linged & , & \begin{tabular}{|l|l|l|l|l|l} 
linver \\
\end{tabular} & & $\operatorname{lin}$ & $565 * * *$ & ling & $322^{* * * *}$ \\
\hline lexpo & & lexpor & & lex & & \begin{tabular}{|l|l|l|l|l} 
lexpot \\
\end{tabular} & & lexpor & $76^{* * * *}$ & lexpor & $5543^{* * * *}$ \\
\hline lvar & 0,00464786 & lpvr & $-0,02544021$ & lger & 0,0805057 & lrqr & $-0,01624644$ & 1rlr & $0,13508214 * * *$ & 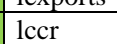 & $-0,11039257^{*}$ \\
\hline
\end{tabular}




\begin{tabular}{|c|c|c|c|c|c|c|c|c|c|c|c|}
\hline ccons & $-4,5828935^{* * * *}$ & _cons & $-4,5349425 * * *$ & cons & $-5,2217945^{* * * *}$ & cons & $-4,8175624 * * *$ & L_cons & $-5,1589435 * * *$ & cons & $-4,213632 * * *$ \\
\hline \multicolumn{12}{|c|}{ PRRN et Faibles institutions } \\
\hline 1ress & $-0,01447299$ & lress & $-0,04374605$ & lress & $-0,03689382$ & lress & 0,01059825 & 1ress & $-0,02901661$ & 1ress & $-0,0656999$ \\
\hline linvest & 0,19797756 *** & linvest & $0,23070843^{* * *}$ & linvest & $0,2312944 * * *$ & linvest & 0,27341196 **** & linvest & $0,20291335^{* * * *}$ & linvest & $0,19693431^{* * * *}$ \\
\hline lexports & $0,41472621 * * *$ & lexports & $0,34809828 * * *$ & lexports & $0,37063541 * * *$ & lexports & $0,30936115^{* * * *}$ & lexports & $0,40152357 * * *$ & lexports & 0,43197737 *** \\
\hline lvar & $-0,07741376 *$ & $1 \mathrm{pvr}$ & $-0,0373352$ & lger & 0,0215268 & lrqr & 0,14783477 *** & 1rlr & $0,05843052 *$ & $1 \mathrm{ccr}$ & 0,03844907 \\
\hline _cons & $-6,2100995^{* * * *}$ & cons & $-5,6593557$ **** & cons & $-6,150141 * * *$ & _cons & $-6,1844077$ **** & _cons & $-6,4053341 * * *$ & cons & $-6,747263 * * *$ \\
\hline \multicolumn{12}{|c|}{ PRRN et Fortes institutions } \\
\hline 1ress & $-0,04897348$ & lress & $-0,06626002$ & lress & $-0,00846462$ & lress & $-0,12792638$ & 1ress & 0,05561503 & 1ress & 0,0115645 \\
\hline linvest & $0,26392374 * * *$ & linvest & $0,14856291 * * *$ & linvest & $0,14732676^{*}$ & linvest & $0,12399543 *$ & linvest & $0,24620315^{* * *}$ & linvest & $0,31620646^{* * * *}$ \\
\hline lexports & $0,33296996^{* * *}$ & lexports & $0,48294907 * * *$ & lexports & $0,60888243 * * *$ & lexports & $0,55619086^{* * *}$ & lexports & $0,43757856^{* * *}$ & lexports & $0,32782751^{* * * *}$ \\
\hline lvar & $0,46830772 *$ & $1 p v r$ & $0,1682107^{*}$ & lger & 0,36873947 **** & lrqr & $0,36123602 * * *$ & 1rlr & 0,15419652 & lccr & $0,27905439^{*}$ \\
\hline _cons & $-7,6506671 * * *$ & cons & $-7,1739507$ **** & cons & $-11,12125 * * *$ & cons & $-9,0078993^{* * * *}$ & cons & $-8,6992787 * * *$ & cons & $-8,0654134 * * *$ \\
\hline
\end{tabular}




\section{Estimation des seuils pour les institutions et les ressources dans les pays d'ASS}

Dans tous les cas, 300 répliques bootstrap ont été utilisées pour tester les effets de seuil dans les différents modèle ( 1,2 et 3 seuils). Les résultats évaluent les seuils dans le cadre des différents modèles. Les tests d'hypothèse sont : $\mathrm{H} 0: \beta 1=\beta 2$ (aucun effet de seuil) et $\mathrm{H1}: \beta 1 \neq \beta 2$ (l'effet de seuil existe). La règle de décision est la suivante : la valeur de la $\mathrm{F}$ statistics est comparée à celle de la valeur critique ; si la F statistics est supérieure, alors on accepte l'hypothèse H1. Ainsi, on conclura qu'il existe une relation non linéaire.

Les résultats montrent qu'il existe une certaine sensibilité entre la nature de la mesure des institutions qui est adoptée et les rentes des ressources naturelles. Ils révèlent, en outre, que lorsque les institutions sont choisies comme variable seuil, seuls l'état de droit et l'efficacité des pouvoirs publics confirment l'existence de la relation non linéaire entre les ressources naturelles et le développement.

Les résultats des travaux confirment l'existence de non-linéarités dans le lien entre institutions et développement.

\section{Estimations des seuils de ressources pour tous les pays}

Un modèle à effets de seuil unique a pu être validé entre les rentes des ressources naturelles et le développement. Le seuil est estimé à 3,14\%. Les résultats montrent que lorsque les rentes des ressources naturelles sont inférieures à ce seuil, toute augmentation de $1 \%$ de ces dernières se traduit par une hausse du PIB par habitant de 0,05\%, alors que lorsqu'elles sont supérieures à ce seuil, toute augmentation de $1 \%$ de ces dernières se traduit par une baisse du PIB par habitant de 0,08\%. Dans le premier cas, les estimations ne sont pas significatives.

Un autre modèle à double seuil a aussi été validé. La relation entre rentes des ressources naturelles et hausse du PIB par habitant suit différents régimes :

- Lorsque les rentes des ressources naturelles sont inférieures à 27,62\% du PIB, l'élasticité avec le PIB par habitant est de $-0,15$

- Lorsque les rentes des ressources naturelles sont comprises entre $13,59 \%$ et $27,62 \%$ du PIB, l'élasticité avec le PIB par habitant est de - 0,10

- Lorsque les rentes des ressources naturelles sont supérieures à 13,59\% du PIB, l'élasticité avec le PIB par habitant est de $-0,06$.

Tableau 3 : Existence des seuils pour les ressources pour tous les pays

\begin{tabular}{|l|l|l|l|}
\hline & 1 seuil & 2 seuils & 3 seuils \\
\hline lvar & & & \\
\hline lpvr & & & \\
\hline lger & & & \\
\hline lrqr & & & \\
\hline lrlr & & & \\
\hline lccr & & & \\
\hline
\end{tabular}


Tableau 4 : Estimation d'un modèle à seuil unique (voix et responssabilité) Threshold estimator (level = 95):

\begin{tabular}{c|ccc}
\hline model & Threshold & Lower & Upper \\
\hline Th-1 & 1.3714 & 1.3471 & 1.4411 \\
\hline
\end{tabular}

Threshold effect test (bootstrap = 300):

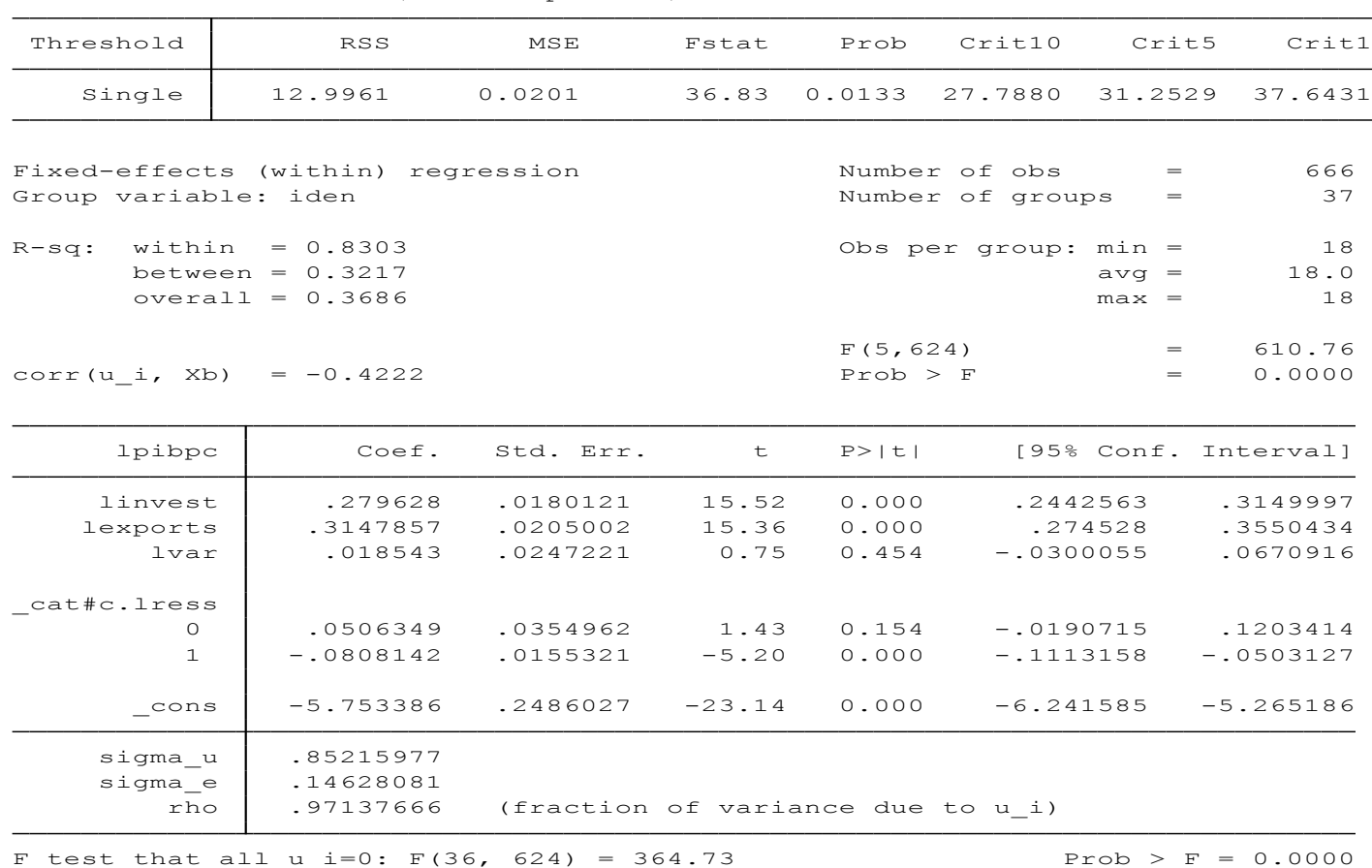

Tableau 5 : Estimation du modèle à double seuil (Stabilité politique et absence de violence)

\begin{tabular}{r|rrr} 
Threshold estimator (level $=95):$ & \\
\hline model & Threshold & Lower & Upper \\
\hline Th-1 & 3.0820 & 2.9159 & 3.1082 \\
Th-21 & 3.3185 & 3.2854 & 3.3777 \\
Th-22 & 2.6095 & 2.5532 & 2.6159 \\
\hline
\end{tabular}

Threshold effect test (bootstrap $=300$
\begin{tabular}{c|ccccccc} 
Threshold & RSS & MSE & Fstat & Prob & Crit10 & Crit5 & Crit1 \\
\hline Single & 13.2442 & 0.0204 & 21.61 & 0.1000 & 21.3663 & 24.3727 & 39.6006 \\
Double & 12.8451 & 0.0198 & 20.13 & 0.0767 & 17.8421 & 22.5352 & 29.5629 \\
\hline
\end{tabular}

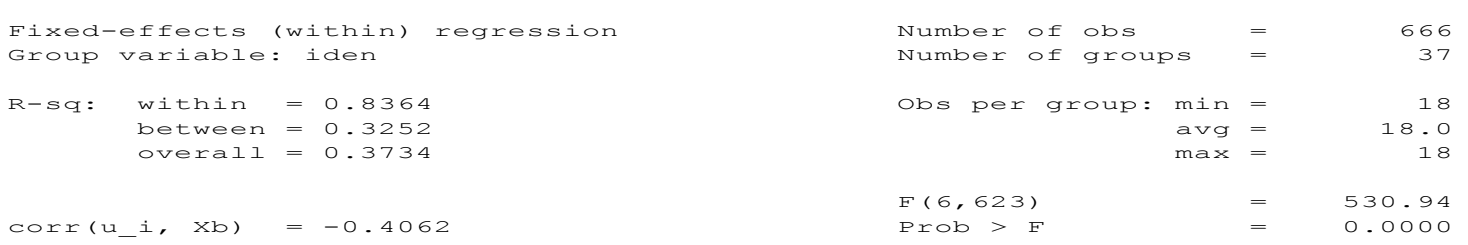

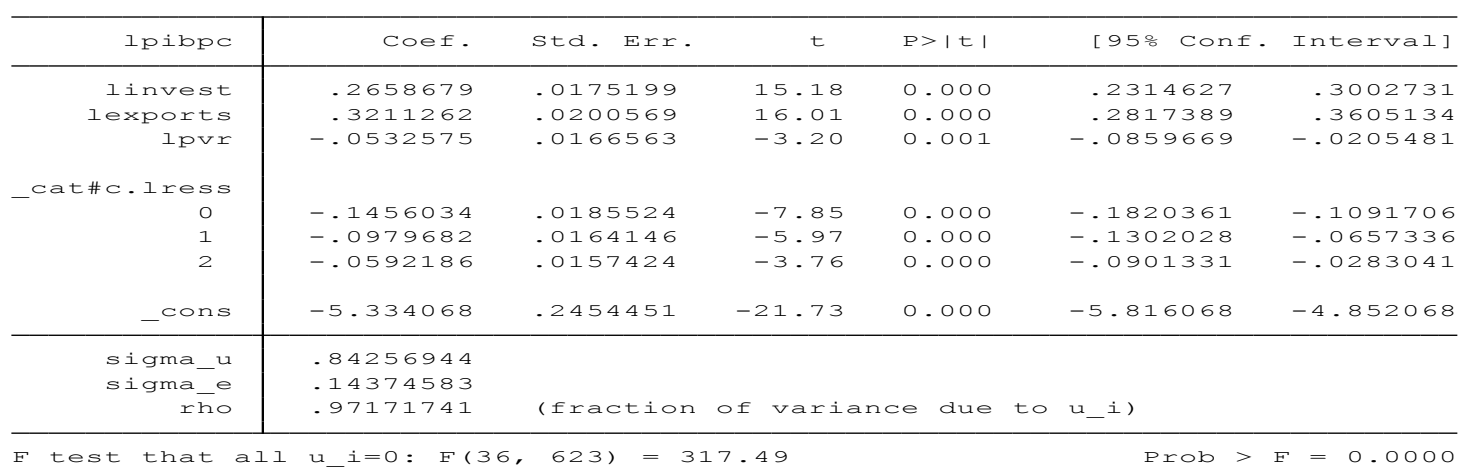




\section{Estimations des seuils des institutions pour tous les pays}

Les résultats montrent que l'impact des institutions sur le développement est positif jusqu'à un certain seuil estimé. Cet impact diminue et devient même négatif suivant la nature de la mesure des institutions.

Tableau 5 : Existence des seuils pour les institutions pour tous les pays

\begin{tabular}{|l|l|l|l|}
\hline & 1 seuil & 2 seuils & 3 seuils \\
\hline lvar & & & \\
\hline lpvr & & & \\
\hline lger & & & \\
\hline lrqr & & & \\
\hline lrlr & & & \\
\hline lccr & & & \\
\hline
\end{tabular}

Légende

\begin{tabular}{c} 
Non concluant \\
$10 \%$ \\
$5 \%$ \\
$1 \%$ \\
\hline
\end{tabular}

Tableau 6 : Estimation du modèle à seuil unique (Efficacité des pouvoirs publics) Threshold estimator (level = 95) :

\begin{tabular}{c|ccc}
\hline model & Threshold & Lower & Upper \\
\hline Th-1 & 2.4115 & 2.3902 & 2.4255 \\
\hline
\end{tabular}

Threshold effect test (bootstrap = 300):

\begin{tabular}{|c|c|c|c|c|c|c|c|}
\hline Threshold & RSS & MSE & Fstat & Prob & Critlo & Crit 5 & Critl \\
\hline Single & 13.1348 & 0.0203 & 29.41 & 0.0833 & 27.0570 & 33.1623 & 57.1744 \\
\hline
\end{tabular}

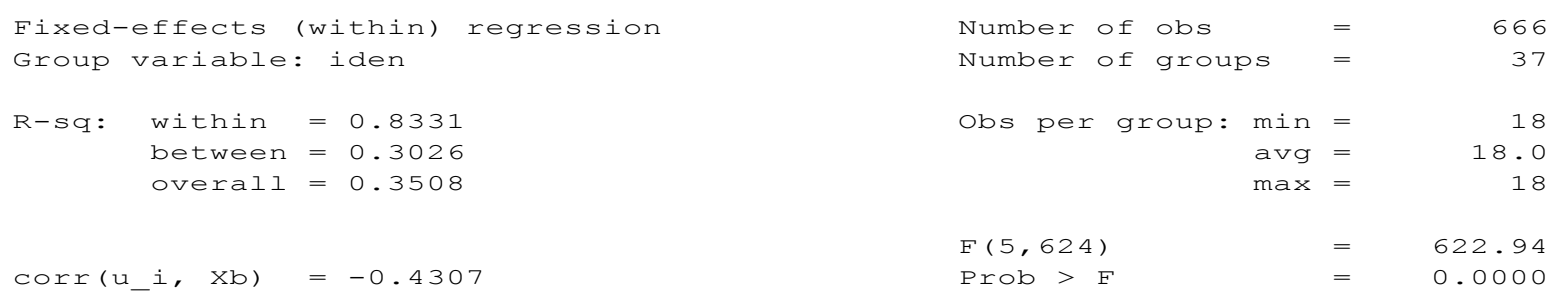

\begin{tabular}{|c|c|c|c|c|c|c|c|}
\hline Ipibpc & Coef. & std. Err. & t & $P>|t|$ & & [95\% Conf. & Interval] \\
\hline Iinvest & .2818758 & .0176703 & 15.95 & 0.000 & & .2471754 & .3165762 \\
\hline lexports & .311427 & .0204113 & 15.26 & O. ० O O & & .2713439 & .3515102 \\
\hline Iress & -.0426973 & .0172669 & -2.47 & 0.014 & & -.0766055 & -.0087891 \\
\hline \multicolumn{8}{|l|}{ cat\#c.lger } \\
\hline 0 & .0256461 & .0172838 & 1.48 & 0.138 & & -.0082954 & .0595877 \\
\hline 1 & -.0154003 & .0174822 & -0.88 & 0.379 & & -.0497315 & .0189308 \\
\hline _cons & -5.776305 & .24877 & $-23 \cdot 22$ & 0.000 & & -6.264832 & -5.287777 \\
\hline sigma_u & .86854159 & & & & & & \\
\hline sigma_e & .14508382 & & & & & & \\
\hline rho & .97285409 & (fraction & of variance & e due & to & $u_{-i}$ ) & \\
\hline
\end{tabular}


Tableau 7 : Estimation du modèle à seuil unique (Etat de droit)

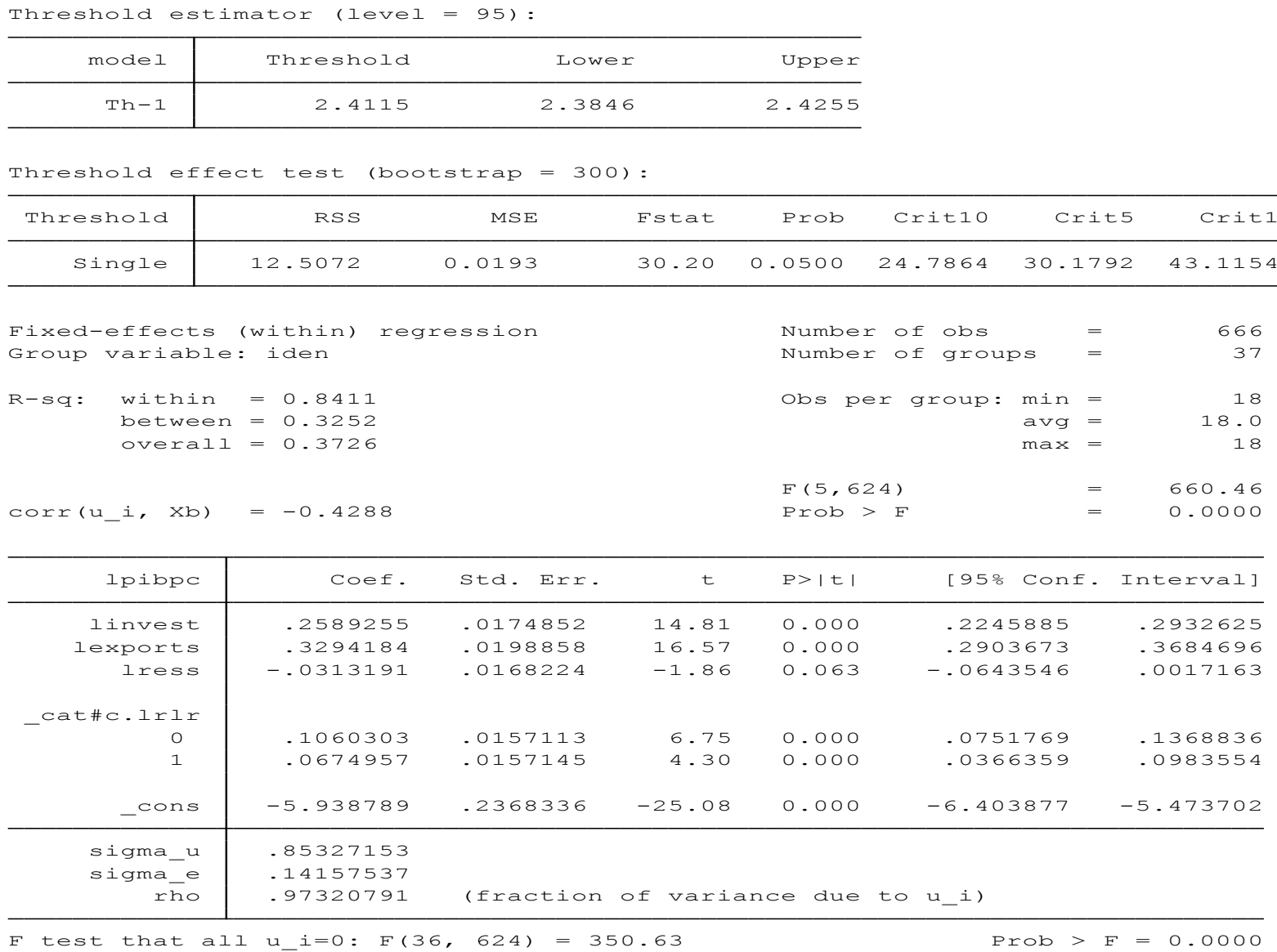

\section{Discussion}

De façon globale, l'impact des ressources naturelles sur le développement en ASS a été faible et négatif sur la période 2002-2019. Aucune différence significative n'est notée entre les pays d'ASS avec de faibles institutions et ceux avec de meilleures institutions, en ce qui concerne l'impact des ressources naturelles sur le développement. Cependant, l'intensité est plus forte dans les pays d'ASS caractérisés par de plus faibles institutions.

Les ressources naturelles contribuent négativement au PIB par habitant, quel que soit le niveau de richesse en ressources. Cependant, cette contribution négative n'est pas significative dans les pays riches en ressources naturelles. De même, l'impact est plus faible dans les pays riches en ressources naturelles.

Les résultats Rahim et al. (2021) vont dans le même sens que ceux trouvés dans le cadre de la présente étude : les rentes des ressources naturelles impactent positivement le développement jusqu'à un certain seuil, au-delà duquel l'impact devient négatif. En effet, Rahim et al. (2021) ont vérifié cette hypothèse et leur conclusion est que la rente plus élevée des ressources naturelles inhibe la croissance économique des pays de leur échantillon. En outre, cette relation non-linéaire a aussi été trouvée par He et al. (2018), pour qui, l'impact des ressources naturelles sur la croissance économique est non linéaire et que la relation entre ces variables présente une courbe en forme de $U$ inversé. Ce résultat signifie que lorsque l'abondance des ressources franchit la valeur seuil, l'effet de la malédiction des ressources se produira. Kalmarzi et al. (2020) ont eu des résultats similaires pour le cas de l'Iran. Selon eux, tant que les revenus pétroliers ont une part inférieure à 16,3\% dans le PIB, les revenus pétroliers ont un effet positif sur la croissance mais après avoir dépassé ce seuil, les rentes pétrolières ont eu un effet négatif et significatif sur la croissance économique. 
Cependant, la malédiction des ressources naturelles n'est pas une fatalité. Bakwena (2012), confirme l'hypothèse selon laquelle les économies riches en ressources ne sont pas destinées à être maudites si elles disposent de bonnes institutions.

Hassan et al. (2019) affirment que la qualité institutionnelle joue un rôle modérateur de l'effet négatif de la richesse pétrolière sur la croissance économique à long terme, tandis qu'à court terme, elle vient renforcer l'effet positif de la richesse pétrolière sur la croissance économique. Ces auteurs ont pu déterminer les seuils de qualité institutionnelle au-delà desquels, la richesse pétrolière améliore la croissance économique, à la fois à long terme et à court terme, pour les pays de leur échantillon.

Veisi (2017) a souligné les problèmes de corruption et de gouvernance comme étant le principal moteur de la malédiction des ressources ; l'auteur s'est appuyé sur l'existence d'une relation négative entre l'abondance des ressources et le contrôle de la corruption dans la littérature. Ses résultats montrent comment la corruption entraîne une malédiction des ressources.

Ainsi, au regard de ces résultats, l'intérêt des recettes issues des ressources naturelles au plan économique devient mitigé et discutable pour certains pays. Tant sur le plan théorique qu'empirique, l'analyse du lien entre recettes issues des ressources naturelles et PIB par habitant ne fait pas consensus. Une richesse en ressources naturelles ne garantit pas automatiquement le développement, mais ne le contraint pas non plus. Avec une prise en charge des institutions, il devient plus clair que les pays peuvent bien tirer parti de la présence des ressources naturelles. De bonnes institutions présentes dans un pays, sont associées à un impact négatif plus faible des ressources naturelles sur le développement.

Les résultats suggèrent que les pays en développement riches en ressources devraient investir dans les institutions de bonne qualité pour espérer profiter de ces ressources. Des mesures adéquates sont nécessaires pour améliorer leur état de qualité institutionnelle, indépendamment du niveau de richesse en ressources naturelles.

\section{Conclusion}

En utilisant des données de panel de 37 pays d'ASS, cette étude a analysé la relation qui existe dans cette zone, entre les recettes issues des ressources naturelles et le développement. Un modèle de régression à effets fixes, à la Hansen (1999) a été utilisé.

Les résultats montrent que les rentes des ressources naturelles ont faiblement, négativement et significativement contribué à l'amélioration du bien-être des populations d'ASS entre 2002 et 2019. Le modèle à effets de seuil unique validé entre les rentes des ressources naturelles et le développement estime un seuil à 3,14\%. Les résultats montrent que lorsque les rentes des ressources naturelles sont inférieures à ce seuil, on est en présence d'une relation positive entre les ressources naturelles et le PIB par habitant (élasticité de $0,05 \%$ ), alors que lorsqu'elles sont supérieures à ce seuil, la relation est négative (élasticité de $-0,08 \%)$.

Il est apparu qu'en présence de meilleures institutions, l'impact négatif des ressources naturelles sur le développement est édulcoré. Ce résultat est utile pour les gestionnaires dans la lutte contre le syndrome hollandais qui affecte plusieurs pays riches en ressources naturelles. Cependant, le niveau du seuil audelà duquel un changement de régime est opéré mérite d'être déterminé pour chaque pays individuellement.

\section{BIBLIOGRAPHIE}

[1] All Answers Ltd. (2018). Resource Rent and Economic Growth_ A Panel Threshold Analysis.pdf. https://ukdiss.com/examples/resource-rent-and-economic-growth.php?vref=1

[2] Balde, M. T. (2020). Natural resources and development: three essays. Thèse Présentée et soutenue publiquement le 17 décembre 2020 pour l'obtention du titre de Docteur és Sciences Economiques pp. 163. 
https://cerdi.uca.fr/medias/fichier/thesefin-mamadou-tanou-balde_1610352303065pdf?ID_FICHE $=122051 \&$ INLINE=FALSE

[3] Canuto, O., \& Daoulas, C. (2019). Natural Wealth and Economic Growth: The Case of Sub-Saharan Africa. 29. https://media.africaportal.org/documents/Natural_wealth_and_economic_growth.pdf

[4] Clootens, N., \& Kirat, D. (2020). Threshold regressions for the resource curse. Environment and Development Economics, 25(6), 583 610. https://doi.org/10.1017/S1355770X20000297

[5] Epo, B. N., \& Nochi Faha, D. R. (2019). Natural Resources, Institutional Quality, and Economic Growth : An African Tale. The European Journal of Development Research, 32(1), 99 128. https://doi.org/10.1057/s41287-019-00222-6

[6] Gylfason, T. (2010). La possession de ressources naturelles est-elle vraiment un privilège ? 42. https://www.imf.org/ /media/Websites/IMF/imported-

events/external/french/np/seminars/2010/afrfin/pdf/_Gylfason2fpdf.ashx\#: :text=Principe\%20presque\%20universel\%2 C\%201a\%20possession,nationales\%20(Wenar\%2C\%202008).

[7] Hassan, A. S., Meyer, D. F., \& Kot, S. (2019). Effect of Institutional Quality and Wealth from Oil Revenue on Economic Growth in Oil-Exporting Developing Countries. Sustainability, 11(13), 3635. https://doi.org/10.3390/su11133635

[8] Hayat, A., \& Tahir, M. (2017). Foreign direct investment, natural resources and economic growth : A threshold model approach. Journal of Economic Studies, 48(5), 929 944. https://doi.org/10.1108/JES-03-2020-0127

[9] He, H., Liu, W., Gao, Q., \& Jiang, L. (2018). Resource Status and Curse Threshold—An Empirical Study Based on Crossnational Panel Data. Proceedings of the 3rd International Conference on Contemporary Education, Social Sciences and Humanities (ICCESSH 2018). 3rd International Conference on Contemporary Education, Social Sciences and Humanities (ICCESSH 2018), Moscow, Russia. https://doi.org/10.2991/iccessh-18.2018.221

[10] Izvorski, I., Coulibaly, S., \& Doumbia, D. (2018). Reinvigorating Growth in Resource-Rich Sub-Saharan Africa. https://doi.org/10.1596/30399

[11] Kalmarzi, H. S., Fattahi, S., \& Soheili, K. (2020). Threshold Effects of Oil Revenues on Iran's Growth Regimes : A Hybrid Threshold Markov Switching Model. Iranian Economic Review, Online First. https://doi.org/10.22059/ier.2020.74559

[12] Kelikume, I. (2018). An Estimation of Inflation Threshold for Africa. International Journal of Management, Economics and Social Sciences, 7(4). https://doi.org/10.32327/IJMESS/7.4.2018.18

[13] Khan, M. A., Khan, M. A., Ali, K., Popp, J., \& Oláh, J. (2020). Natural Resource Rent and Finance : The Moderation Role of Institutions. Sustainability, 12(9), 3897. https://doi.org/10.3390/su12093897

[14] Moshiri, S., \& Hayati, S. (2017). Natural Resources, Institutions Quality, and Economic Growth; A Cross-Country Analysis. 33. ftp://80.66.179.253/eut/journl/20173-11.pdf

[15] Ndoricimpa, A. (2017). Threshold Effects of Inflation on Economic Growth in Africa : Evidence from a Dynamic Panel Threshold Regression $\quad$ Approach. 38. https://www.afdb.org/fileadmin/uploads/afdb/Documents/Publications/WPS_No_249_Threshold_Effects_of_Inflation_o n_Economic_Growth_in_Africa.pdf

[16] Rahim, S., Murshed, M., Umarbeyli, S., Kirikkaleli, D., Ahmad, M., Tufail, M., \& Wahab, S. (2021). Do natural resources abundance and human capital development promote economic growth? A study on the resource curse hypothesis in Next Eleven countries. Resources, Environment and Sustainability, 4, 100018. https://doi.org/10.1016/j.resenv.2021.100018

[17] Sarmidi, T., Hook Law, S., \& Jafari, Y. (2011). Resource Curse: New Evidence on the Role of Institutions. International Economic Journal, 28(1), 191 206. https://doi.org/10.1080/10168737.2013.787110

[18] Song, M., Liu, B., Zhang, J., \& Keqing, L. (2021). Mineral Economic Regionalization in the Context of Resource Crisis and Sustained Economic Growth. IOP Conference Series: Earth and Environmental Science, 781(3), 032061. https://doi.org/10.1088/1755-1315/781/3/032061

[19] Sun, Y., Sun, H., Chen, L., Taghizadeh-Hesary, F., \& Zhao, G. (2020). Impact of natural-resource dependence on foreign contracting projects of China: A spatial panel threshold approach. PLOS ONE, 15(6), e0234057. https://doi.org/10.1371/journal.pone.0234057

[20] Veisi, M. (2017). Essays on the links between Natural Resources, Corruption, Taxation and Economic Growth.pdf [University of Manchester]. https://www.research.manchester.ac.uk/portal/files/55559015/FULL_TEXT.PDF 